\title{
Reentrant Phase Transitions and Triple Points of Topological AdS Black Holes in Born-Infeld-Massive Gravity
}

\author{
Ming Zhang, ${ }^{1,2}$ De-Cheng Zou, ${ }^{3}$ and Rui-Hong Yue ${ }^{3}$ \\ ${ }^{1}$ Faculty of Science, Xian Aeronautical University, Xian 710077, China \\ ${ }^{2}$ National Joint Engineering Research Center of Special Pump System Technology, Xian 710077, China \\ ${ }^{3}$ Center for Gravitation and Cosmology, College of Physical Science and Technology, Yangzhou University, Yangzhou 225009, China
}

Correspondence should be addressed to Ming Zhang; shakellar@126.com

Received 6 July 2017; Revised 13 September 2017; Accepted 12 October 2017; Published 6 November 2017

Academic Editor: Hai-Qing Zhang

Copyright (c) 2017 Ming Zhang et al. This is an open access article distributed under the Creative Commons Attribution License, which permits unrestricted use, distribution, and reproduction in any medium, provided the original work is properly cited. The publication of this article was funded by SCOAP ${ }^{3}$.

\begin{abstract}
Motivated by recent developments of black hole thermodynamics in de Rham, Gabadadze, and Tolley (dRGT) massive gravity, we study the critical behaviors of topological Anti-de Sitter (AdS) black holes in the presence of Born-Infeld nonlinear electrodynamics. Here the cosmological constant appears as a dynamical pressure of the system and its corresponding conjugate quantity is interpreted as thermodynamic volume. This shows that, besides the Van der Waals-like SBH/LBH phase transitions, the socalled reentrant phase transition (RPT) appears in four-dimensional space-time when the coupling coefficients $c_{i} m^{2}$ of massive potential and Born-Infeld parameter $b$ satisfy some certain conditions. In addition, we also find the triple critical points and the small/intermediate/large black hole phase transitions for $d=5$.
\end{abstract}

\section{Introduction}

Einstein's General Relativity (GR), which describes that the graviton is a massless spin-2 particle, helped us to understand the dynamics of the Universe [1-3]. However, there are some fundamental issues, such as the hierarchy problem in particle physics, the old cosmological constant problem, and the origin of late-time acceleration of the Universe, that still exist in GR [4]. One of the alternating theories of gravity is known as a massive gravity, where mass terms are added to the GR action. A graviton mass has the advantage to potentially provide a theory of dark energy which could explain the present day acceleration of our Universe [5]. On the other hand, since the quantum theory of massless gravitons is nonrenormalizable, a natural question is whether one can build a self-consistent gravity theory if the graviton is massive. The first attempt toward constructing the theory of massive gravity was done by Fierz and Pauli (FP) [6]. With the quadratic order, the FP mass term is the only ghost-free term describing a gravity theory with five degrees of freedom [7]. However, due to the existence of the van Dam-Veltman-Zakharov (vDVZ) discontinuity, this theory cannot recover linearized Einstein gravity in the limit of vanishing graviton mass $[8,9]$.

In particular, Vainshtein [10] proposed that the linear massive gravity can be recovered to GR through the "Vainshtein Mechanism" at small scales by including nonlinear terms in the massive gravity action. Nevertheless, it usually brings various instabilities for the gravitational theories on the nonlinear level by adding generic mass terms, since this model suffers from a pathology called a "Boulware-Deser" (BD) ghost. Later, a new nonlinear massive gravity theory was proposed by de Rham, Gabadadze, and Tolley (dRGT) [1113], where the BD ghost [14] was eliminated by introducing higher order interaction terms in the action. Then, Vegh et al. $[15,16]$ constructed a nontrivial black hole solution with a Ricci flat horizon in four-dimensional dRGT massive gravity. The spherically symmetric solutions were also addressed in [17-19]; the corresponding charged black hole solution was found in [20,21].

Recent development on the thermodynamics of black holes in extended phase space shows that the cosmological constant can be interpreted as the thermodynamic pressure 
and treated as a thermodynamic variable in its own right $[22,23]:$

$$
P=-\frac{\Lambda}{8 \pi}
$$

in the geometric units $G_{N}=\hbar=c=1$. Such operation assumes that gravitational theories including different values of the cosmological constants fall in the same class, with unified thermodynamic relations. For black hole thermodynamics, the variation of the cosmological constant ensures the consistency between the first law of black hole thermodynamics and the Smarr formula. Moreover, the classical theory of gravity may be an effective theory which follows from a yet unknown fundamental theory, in which all the presently "physical constants" are actually moduli parameters that can run from place to place in the moduli space of the fundamental theory. Since the fundamental theory is yet unknown, it is more reasonable to consider the extended thermodynamics of gravitational theories involving only a single action, and then all variables will appear in the thermodynamical relations. In the extended phase space, the charged AdS black hole black hole admits a more direct and precise coincidence between the first-order small/large black holes $(\mathrm{SBH} / \mathrm{LBH})$ phase transition and the Van der Waals liquid-gas phase transition, and both systems share the same critical exponents near the critical point [24]. More discussions in various gravity theories can be found in [2546]. Recently, some investigations for thermodynamics of AdS black holes have been also generalized to the extended phase space in the dRGT massive gravity [47-50], which show the Van der Waals-like SBH/LBH phase transition in the charged topological AdS black holes. In addition, the deep relation between the dynamical perturbation and the Van der Waals-like SBH/LBH phase transition in the fourdimensional dRGT massive gravity has been also recovered in [51]. In particular, for neutral AdS black holes in all $d \geq 6$ dimensional space-time, there exist peculiar behaviors of intermediate/small/large black hole phase transitions reminiscent of reentrant phase transitions (RPTs) when the coupling coefficients $c_{i} m^{2}$ of massive potential satisfy some certain conditions [52]. A system undergoes an RPT if a monotonic variation of any thermodynamic quantity results in two (or more) phase transitions such that the final state is macroscopically similar to the initial state. The RPT is usually observed in multicomponent fluid systems, ferroelectrics, gels, liquid crystals, and binary gases [53].

In Maxwell's electromagnetic field theory, a point-like charge which allowed a singularity at the charge position usually brings about infinite self-energy. In order to overcome this problem, Born and Infeld [54] and Hoffmann [55] introduced Born-Infeld electromagnetic field to solve infinite self-energy problem by imposing a maximum strength of the electromagnetic field. In addition, BI type effective action arises in an open superstring theory and D-branes are free of physical singularities. In recent two decades, exact solutions of gravitating black objects in the presence of BI theory have been vastly investigated. In the extended phase space, $[56,57]$ recovered the RPT in the four-dimensional EinsteinBorn-Infeld AdS black hole with spherical horizon. However, for the higher-dimensional Einstein-Born-Infeld AdS black holes, there is no RPT. What about AdS black holes in the Born-Infeld-massive gravity? In this paper, we will generalize the discussion to topological AdS black holes for $d=4$ and 5 in the Born-Infeld-massive gravity.

This paper is organized as follows. In Section 2, we review the thermodynamics of Born-Infeld-massive black holes in the extended phase space. In Section 3, we study the critical behaviors of four- and five-dimensional topological AdS black holes in context of $P-V$ criticality and phase diagrams. We end the paper with conclusions and discussions in Section 4.

\section{Thermodynamics of $d$-Dimensional Born-Infeld AdS Black Holes}

We start with the action of $d$-dimensional massive gravity in presence of Born-Infeld field [58]:

$$
\begin{aligned}
\mathscr{I} & =\frac{1}{16 \pi} \int d^{d} x \sqrt{-g}[R-2 \Lambda+\mathscr{L}(\mathscr{F}) \\
& \left.+m^{2} \sum_{i=1}^{4} c_{i} \mathscr{U}_{i}(g, f)\right],
\end{aligned}
$$

where the last four terms are the massive potential associated with graviton mass, $c_{i}$ are the negative constants [21], and $f$ is a fixed rank- 2 symmetric tensor. Moreover, $\mathscr{U}_{i}$ are symmetric polynomials of the eigenvalues of the $d \times d$ matrix $\mathscr{K}_{v}^{\mu} \equiv$ $\sqrt{g^{\mu \alpha} f_{\alpha \nu}}$ :

$$
\begin{aligned}
\mathscr{U}_{1}= & {[\mathscr{K}], } \\
\mathcal{U}_{2}= & {[\mathscr{K}]^{2}-\left[\mathscr{K}^{2}\right], } \\
\mathcal{U}_{3}= & {[\mathscr{K}]^{3}-3[\mathscr{K}]\left[\mathscr{K}^{2}\right]+2\left[\mathscr{K}^{3}\right], } \\
\mathcal{U}_{4}= & {[\mathscr{K}]^{4}-6\left[\mathscr{K}^{2}\right][\mathscr{K}]^{2}+8\left[\mathscr{K}^{3}\right][\mathscr{K}]+3\left[\mathscr{K}^{2}\right]^{2} } \\
& -6\left[\mathscr{K}^{4}\right] .
\end{aligned}
$$

The square root in $\mathscr{K}$ is understood as the matrix square root, that is, $(\sqrt{A})^{\mu}{ }_{\nu}(\sqrt{A})_{\lambda}{ }^{\nu}=A^{\mu}{ }_{\lambda}$, and the rectangular brackets denote traces $[\mathscr{K}]=\mathscr{K}^{\mu}{ }_{\mu}$. In addition, $b$ is the Born-Infeld parameter and $\mathscr{L}(\mathscr{F})$ with

$$
\mathscr{L}(\mathscr{F})=4 b^{2}\left(1-\sqrt{1+\frac{F^{\mu \nu} F_{\mu \nu}}{2 b^{2}}}\right) .
$$

In the limit $b \rightarrow \infty$, it reduces to the standard Maxwell field $\mathscr{L}(\mathscr{F})=-F^{\mu \nu} F_{\mu \nu}+\mathcal{O}\left(F^{4}\right)$. If taking $b=0, \mathscr{L}(\mathscr{F})$ disappears.

Consider the metric of $d$-dimensional space-time in the following form:

$$
d s^{2}=-f(r) d t^{2}+\frac{1}{f(r)} d r^{2}+r^{2} h_{i j} d x^{i} d x^{j},
$$

where $h_{i j} d x^{i} d x^{j}$ is the line element for an Einstein space with constant curvature $(d-2)(d-3) k$. The constant $k$ 
characterizes the geometric property of hypersurface, which takes values $k=0$ for flat, $k=-1$ for negative curvature, and $k=1$ for positive curvature, respectively.

By using the reference metric [21]

$$
f_{\mu \nu}=\operatorname{diag}\left(0,0, c_{0}^{2} h_{i j}\right)
$$

with a positive constant $c_{0}$, we can obtain

$$
\begin{aligned}
& \mathscr{U}_{1}=\frac{(d-2) c_{0}}{r}, \\
& \mathcal{U}_{2}=\frac{(d-2)(d-3) c_{0}^{2}}{r^{2}}, \\
& \mathcal{U}_{3}=\frac{(d-2)(d-3)(d-4) c_{0}^{3}}{r^{3}}, \\
& \mathcal{U}_{4}=\frac{(d-2)(d-3)(d-4)(d-5) c_{0}^{4}}{r^{4}} .
\end{aligned}
$$

Obviously, the terms related to $c_{3}$ and $c_{4}$ only appear in the black hole solutions for $d \geq 5$ and $d \geq 6$, respectively [21].

In addition, the electromagnetic field tensor in $d$ dimensions is given by $F_{\mathrm{tr}}=\sqrt{d_{2} d_{3} /(1+\Gamma)}\left(q / r^{d_{2}}\right)$, and the metric function $f(r)$ is obtained as follows [58]:

$$
\begin{aligned}
f(r)= & k-\frac{m_{0}}{r^{d_{3}}}+\frac{\left(4 b^{2}-2 \Lambda\right)}{d_{1} d_{2}} r^{2}-\frac{4 b^{2} r^{2}}{d_{1} d_{2}} \sqrt{1+\Gamma} \\
& +\frac{4 d_{2} q^{2}}{d_{1} r^{2 d_{3}}} \mathscr{H} \\
& +m^{2} c_{0}\left(\frac{c_{1} r}{d_{2}}+c_{0} c_{2}+\frac{d_{3} c_{0}^{2} c_{3}}{r}+\frac{d_{3} d_{4} c_{0}^{3} c_{4}}{r^{2}}\right),
\end{aligned}
$$

where $d_{i}=d-i$ and

$$
\begin{gathered}
\Gamma=\frac{d_{2} d_{3} q^{2}}{b^{2} r^{2 d_{2}}} \\
\mathscr{H}={ }_{2} F_{1}\left[\frac{1}{2}, \frac{d_{3}}{2 d_{2}}, \frac{3 d_{7 / 3}}{2 d_{2}},-\Gamma\right] .
\end{gathered}
$$

Moreover, $m_{0}$ and $q$ are related to the mass $M$ and charge $Q$ of black holes as

$$
\begin{aligned}
& Q=\frac{\sqrt{d_{2} d_{3}} \Sigma_{k}}{4 \pi} q, \\
& M=\frac{d_{2} \Sigma_{k}}{16 \pi} m_{0},
\end{aligned}
$$

where $\Sigma_{k}$ represents the volume of constant curvature hypersurface described by $h_{i j} d x^{i} d x^{j}$. The electromagnetic potential difference $(\Phi)$ between the horizon and infinity reads as $\Phi=$ $\sqrt{d_{2} / d_{3}}\left(q / r_{+}^{d_{3}}\right) \mathscr{H}_{+}$.
Then the mass $M$ of the Born-Infeld AdS black hole for massive gravity is given by

$$
\begin{aligned}
M & =\frac{d_{2} \Sigma_{k} r_{+}^{d_{3}}}{16 \pi}\left[k+\frac{16 \pi P}{d_{1} d_{2}} r_{+}^{2}+\frac{4 b^{2} r_{+}^{2}}{d_{1} d_{2}}\left(1-\sqrt{1+\Gamma_{+}}\right)\right. \\
& +\frac{4 d_{2} q^{2}}{d_{1} r_{+}^{2 d_{3}}} \mathscr{H}_{+} \\
& \left.+m^{2}\left(\frac{c_{0} c_{1} r_{+}}{d_{2}}+c_{0}^{2} c_{2}+\frac{d_{3} c_{0}^{3} c_{3}}{r_{+}}+\frac{d_{3} d_{4} c_{0}^{4} c_{4}}{r_{+}^{2}}\right)\right],
\end{aligned}
$$

in terms of the horizon radius $r_{+}$. Due to existence of the pressure in obtained relation for total mass of the black holes, here the black hole mass $M$ can be considered as the enthalpy $H$ rather than the internal energy of the gravitational system [59].

In addition, the Hawking temperature which is related to the definition of surface gravity on the outer horizon $r_{+}$can be obtained as

$T$

$$
\begin{aligned}
= & \frac{d_{3} k}{4 \pi r_{+}}+\frac{4 r_{+}}{d_{2}} P+\frac{b^{2} r_{+}}{d_{2} \pi}\left(1-\sqrt{1+\Gamma_{+}}\right) \\
& +\frac{m^{2} c_{0}}{4 \pi}\left(c_{1}+\frac{d_{3} c_{0} c_{2}}{r_{+}}+\frac{d_{3} d_{4} c_{0}^{2} c_{3}}{r_{+}^{2}}+\frac{d_{3} d_{4} d_{5} c_{0}^{3} c_{4}}{r_{+}^{3}}\right),
\end{aligned}
$$

and the entropy $S$ of the Born-Infeld AdS black hole reads as

$$
S=\frac{\Sigma_{k}}{4} r_{+}^{d_{2}}
$$

It is easy to check that those thermodynamic quantities obey the (extended phase space) first law of black hole thermodynamics:

$$
\begin{aligned}
d H= & T d S+V d P+\mathfrak{B} d b+\frac{c_{0} m^{2} \Sigma_{k} r_{+}^{d_{2}}}{16 \pi} d c_{1} \\
& +\frac{d_{2} c_{0}^{2} m^{2} \Sigma_{k} r_{+}^{d_{3}}}{16 \pi} d c_{2}+\frac{d_{2} d_{3} c_{0}^{3} m^{2} \Sigma_{k} r_{+}^{d_{4}}}{16 \pi} d c_{3} \\
& +\frac{d_{2} d_{3} d_{4} c_{0}^{4} m^{2} \Sigma_{k} r_{+}^{d_{5}}}{16 \pi} d c_{4},
\end{aligned}
$$

where $\mathfrak{B}$, which is a quantity conjugate to $b$, is called the "Born-Infeld vacuum polarization":

$$
\begin{aligned}
\mathfrak{B} & =\left.\left(\frac{\partial H}{\partial b}\right)\right|_{\left(S, P, c_{1}, c_{2}, c_{3}, c_{4}\right)} \\
& =\frac{\Sigma_{k} b r_{+}^{d_{1}}}{2 d_{1} \pi}\left(1-\sqrt{1+\Gamma_{+}}\right)+\frac{d_{2} d_{3} \Sigma_{k}}{4 \pi d_{1} b} \frac{\mathscr{H}_{+} q^{2}}{r_{+}^{d_{3}}},
\end{aligned}
$$

and the thermodynamic volume $V$ [60], which is the corresponding conjugate quantity of $P$, can be written as

$$
V=\frac{\sum_{k} r_{1}^{d_{1}}}{d_{1}} .
$$


The behavior of free energy $G$ is important to determine the thermodynamic phase transition in the canonical ensemble. We can calculate the free energy from the thermodynamic relation:

$$
\begin{aligned}
G= & H-T S \\
= & \frac{r_{+}^{d_{1}}}{d_{1} d_{2}}\left(P+\frac{b^{2}}{4 \pi} \sqrt{1+\Gamma_{+}}\right)+\frac{d_{2}^{2} q^{2} \mathscr{H}_{+}}{2 \pi d_{1} r_{+}^{d_{3}}}+\frac{r_{+}^{d_{3}}}{16 \pi} \\
& +\frac{m^{2} c_{0}^{2} r_{+}^{d_{5}}}{16 \pi}\left(c_{2} r_{+}^{2}+2 d_{3} c_{0} c_{3} r_{+}+3 d_{3} d_{4} c_{0}^{2} c_{4}\right) .
\end{aligned}
$$

\section{Phase Transitions of Topological AdS Black Holes in Born-Infeld-Massive Gravity}

For further convenience, we denote

$$
\begin{aligned}
\widehat{T} & =T-\frac{c_{0} c_{1} m^{2}}{4 \pi}, \\
W_{2} & =-\frac{k+c_{0}^{2} c_{2} m^{2}}{8 \pi}, \\
W_{3} & =-\frac{c_{0}^{3} c_{3} m^{2}}{8 \pi}, \\
W_{4} & =-\frac{c_{0}^{4} c_{4} m^{2}}{8 \pi},
\end{aligned}
$$

where $\widehat{T}$ denotes the shifted temperature and can be negative according to the value of $c_{0} c_{1} m^{2}$. Then, the equation of state of the black hole can be obtained from (12):

$$
\begin{aligned}
P & =\frac{d_{2}}{4 r_{+}}\left[\widehat{T}+\frac{2 d_{3} W_{2}}{r_{+}}+\frac{2 d_{3} d_{4} W_{3}}{r_{+}^{2}}+\frac{2 d_{3} d_{4} d_{5} W_{4}}{r_{+}^{3}}\right. \\
& \left.-\frac{b^{2} r_{+}}{d_{2} \pi}\left(1-\sqrt{1+\Gamma_{+}}\right)\right] .
\end{aligned}
$$

To compare with the VdW fluid equation, we can translate the "geometric" equation of state to physical one by identifying the specific volume $v$ of the fluid with the horizon radius of the black hole as $v=4 r_{+} / d_{2}$. Evidently, the specific volume $v$ is proportional to the horizon radius $r_{+}$; therefore we will just use the horizon radius in the equation of state for the black hole hereafter in this paper.

We know that the critical point occurs when $P$ has an inflection point:

$$
\left.\frac{\partial P}{\partial r_{+}}\right|_{\widehat{T}=\widehat{T}_{c}, r_{+}=r_{c}}=\left.\frac{\partial^{2} P}{\partial r_{+}^{2}}\right|_{\widehat{T}=\widehat{T}_{c}, r_{+}=r_{c}}=0,
$$

where the subscript stands for the quantities at the critical point. The critical shifted temperature is obtained as

$$
\begin{aligned}
\widehat{T}_{c}= & -\frac{2 d_{3}}{r_{c}}\left(2 w_{2}+\frac{3 d_{4} W_{3}}{r_{c}}+\frac{4 d_{4} d_{5} W_{4}}{r_{c}^{2}}\right) \\
& -\frac{d_{2} d_{3} q^{2}}{\pi r_{c}^{2 d_{5 / 2}}}\left(1+\Gamma_{+}\right)^{-1 / 2},
\end{aligned}
$$

and the equation for critical horizon radius $r_{c}$ is given by

$$
\begin{aligned}
F\left(r_{c}\right)= & 6 d_{4} d_{5} W_{4}+3 d_{4} W_{3} r_{c}+W_{2} r_{c}^{2} \\
& +\frac{d_{5 / 2} d_{2} q^{2}}{2 \pi r_{c}^{2 d_{4}}}\left(1+\Gamma_{+}\right)^{-1 / 2} \\
& -\frac{d_{3} d_{2}^{3} q^{4}}{4 \pi b^{2} r_{c}^{4 d_{3}}}\left(1+\Gamma_{+}\right)^{-3 / 2}=0
\end{aligned}
$$

For later discussions, it is convenient to rescale some quantities in the following way:

$$
\begin{aligned}
W_{2} & =q^{2 /(d-2)} \cdot b^{2(d-3) /(d-2)} w_{2}, \\
W_{3} & =q^{3 /(d-2)} \cdot b^{(2 d-7) /(d-2)} w_{3}, \\
W_{4} & =q^{4 /(d-2)} \cdot b^{2(d-4) /(d-2)} w_{4}, \\
r_{+} & =\left(\frac{q}{b}\right)^{1 /(d-2)} \cdot x, \\
P & =b^{2} \cdot p, \\
\widehat{T} & =q^{1 /(d-2)} \cdot b^{(2 d-5) /(d-2)} \cdot t, \\
G & =q^{(d-1) /(d-2)} \cdot b^{-2 /(d-2)} \Sigma_{k} \cdot g .
\end{aligned}
$$

In terms of quantities above, (19), (21), and (22) can be written as

$$
\begin{aligned}
p= & \frac{d_{2}}{4 x}\left[t+\frac{2 d_{3} w_{2}}{x}+\frac{2 d_{3} d_{4} w_{3}}{x^{2}}+\frac{2 d_{3} d_{4} d_{5} w_{4}}{x^{3}}\right. \\
& \left.-\frac{x}{d_{2} \pi}\left(1-\sqrt{1+\frac{d_{2} d_{3}}{x^{2 d_{2}}}}\right)\right], \\
t_{c}= & -\frac{2 d_{3}}{x_{c}}\left(2 w_{2}+\frac{3 d_{4} w_{3}}{x_{c}}+\frac{4 d_{4} d_{5} w_{4}}{x_{c}^{2}}\right)-\frac{d_{2} d_{3}}{\pi x_{c}^{2 d_{5 / 2}}}(1 \\
& \left.+\frac{d_{2} d_{3}}{x_{c}^{2 d_{2}}}\right)^{-1 / 2}, \quad \\
F & \left(x_{c}\right)=6 d_{4} d_{5} w_{4}+3 d_{4} w_{3} x_{c}+w_{2} x_{c}^{2}+\frac{d_{2} d_{5 / 2}}{2 \pi x_{c}^{2 d_{4}}}(1 \\
& \left.+\frac{d_{2} d_{3}}{x_{c}^{2 d_{2}}}\right)^{-1 / 2}-\frac{d_{3} d_{2}^{3}}{4 \pi x_{c}^{4 d_{3}}}\left(1+\frac{d_{2} d_{3}}{x_{c}^{2 d_{2}}}\right)^{-3 / 2}=0,
\end{aligned}
$$

where $x_{c}$ denotes the critical value of $x$. For arbitrary parameter $d$, it is hard to obtain the exact solution of (26).

In what follows we shall specialize to $d=4$ and 5 and then perform a detailed study of the thermodynamics of these black holes. 

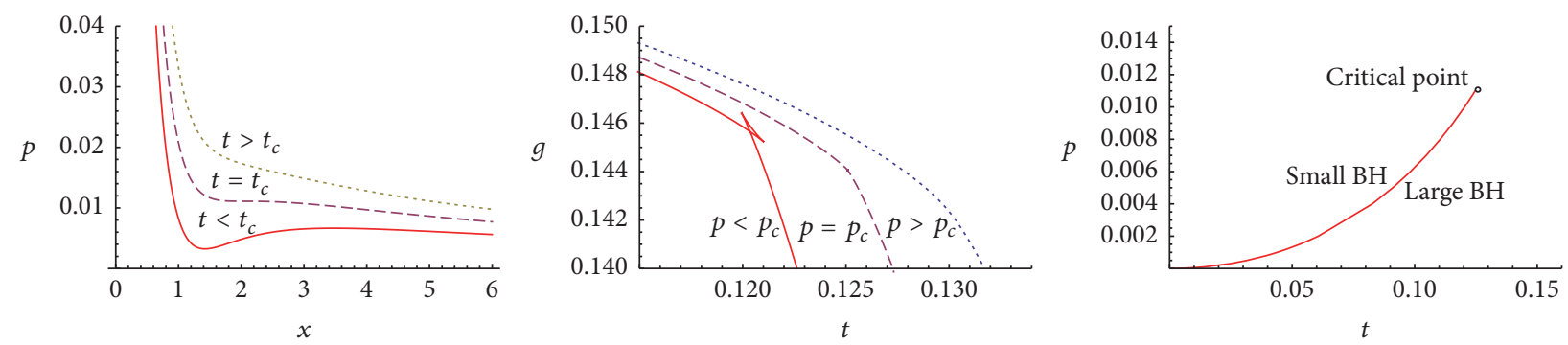

FIGURE 1: Born-Infeld AdS black holes for $d=4$ and $w_{2}=-0.1$. There is one critical point, which corresponds to VdW-like SBH/LBH phase transition when $t<t_{c}$. Here the critical pressure and temperature read $p=p_{c} \approx 0.011986$ and $t=t_{c} \approx 0.125087$, respectively.

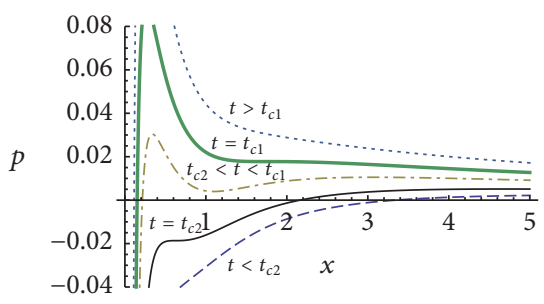

(a)

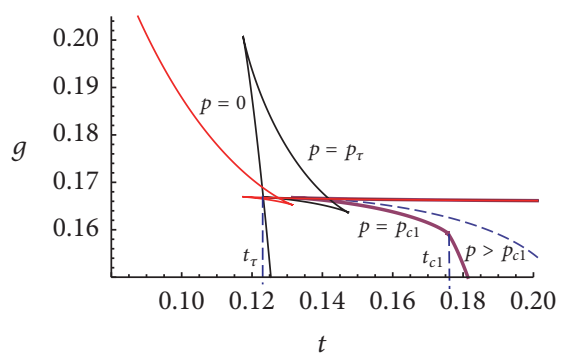

(b)

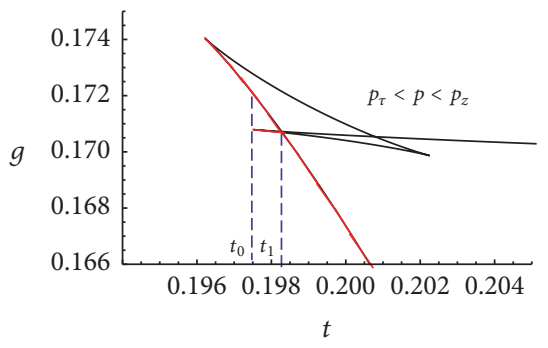

(c)

Figure 2: Born-Infeld AdS black holes for $d=4$ and $w_{2}=-0.124$. (a) The $p-x$ diagram shows the existence of two critical points, one at positive pressure $p_{c 1} \approx 0.0177545$, the other at negative pressure $p_{c 2} \approx-0.0084877$. (b) The Gibbs free energy shows one physical (with positive pressure) critical point and the corresponding first-order SBH/LBH phase transition, occurring for $t \in\left(t_{\tau}, t_{c 1}\right)$ and $p \in\left(p_{\tau}\right.$, $\left.p_{c 1}\right)$. (c) There is a reentrant phase transition (RPT) corresponding to the zeroth-order phase transition at $t=t_{0}$ followed by a first-order VdW-like $\mathrm{SBH} / \mathrm{LBH}$ phase transition at the intersection $t=t_{1}$ with the swallowtail structure.

3.1. $P-V$ Criticality for $d=4$. For $d=4$, (26) will reduce to the cubic equation:

$$
F(y)=y^{3}-\frac{3 y}{4}-\frac{\pi w_{2}}{2}=0
$$

with $y=\left(x_{c}^{4}+2\right)^{-1 / 2}$.

Depending on different values of $w_{2},(27)$ admits one or more positive real roots for $x$, which can be also reflected by

$$
\frac{\partial F(y)}{\partial y}=3 y^{2}-\frac{3}{4}
$$
by

When $\left|w_{2}\right| \leq 1 / 2 \pi$, three real roots occur, which are given

$$
y_{i}=\cos \left(\frac{1}{3} \arccos \left(2 \pi w_{2}\right)-\frac{2 \pi i}{3}\right), \quad i=0,1,2 .
$$

Moreover, in order that $x_{c}=\left(1 / y^{2}-2\right)^{1 / 4}$ be positive, we require an additional constraint $|y| \leq 1 / \sqrt{2}$. Then, we have $y_{0}>0$ in case of $-1 / 2 \pi \leq w_{2} \leq-1 / \sqrt{8} \pi$, and $y_{1}>0$ in the region of $-1 / 2 \pi \leq w_{2} \leq 0$, while the solution $y_{2}$ is always negative.

Now by inserting solutions of $y_{0}$ and $y_{1}$ into (24) and (25), we analyze the critical behaviors. Notice that analytic methods cannot be applied in our analysis because of the complexity of the Gibbs free energy and equation of state, we resort to graphical and numerical methods.
(1) $w_{2} \in(-1 / \sqrt{8} \pi, 0)$. As shown in Figure 1 , the $p-x$ diagram displays that the dashed curve represents critical isotherm at $t=t_{c}$ and the dotted and solid curves correspond to $t>t_{c}$ and $t<t_{c}$, respectively. In the $g-t$ diagram, the solid curve represents $p<$ $p_{c}$, the dotted curve correspond to $p>p_{c}$, and the dashed curve is for $p=p_{c}$. We observe standard swallowtail behavior. Moreover, the $p-t$ diagram shows the coexistence line of the first-order phase transition terminating at a critical point. These plots are analogous to typical behavior of the liquid-gas phase transition of the Van der Waals fluid.

(2) $w_{2} \in(-0.132795,-1 / \sqrt{8} \pi)$, there only exist one physical (with positive pressure) critical point and the corresponding VdW-like SBH/LBH phase transition, which occurs for the pressures $p \in\left(p_{\tau}, p_{c 1}\right)$ and temperatures $t \in\left(t_{\tau}, t_{c 1}\right)$; see Figure 2 . For the $p-t$ diagram in Figure 3, three separate phases of black holes emerge in the region of $p_{\tau}<p \leq p_{z}<p_{c 1}$ : intermediate black holes (IBH) (on the left), small (on the middle), and large (on the right), where small and large black holes are separated by the SBH/LBH phase transition, but the intermediate and small are separated by a finite jump in $g$, which is so-called zeroth-order phase transition [61]. 

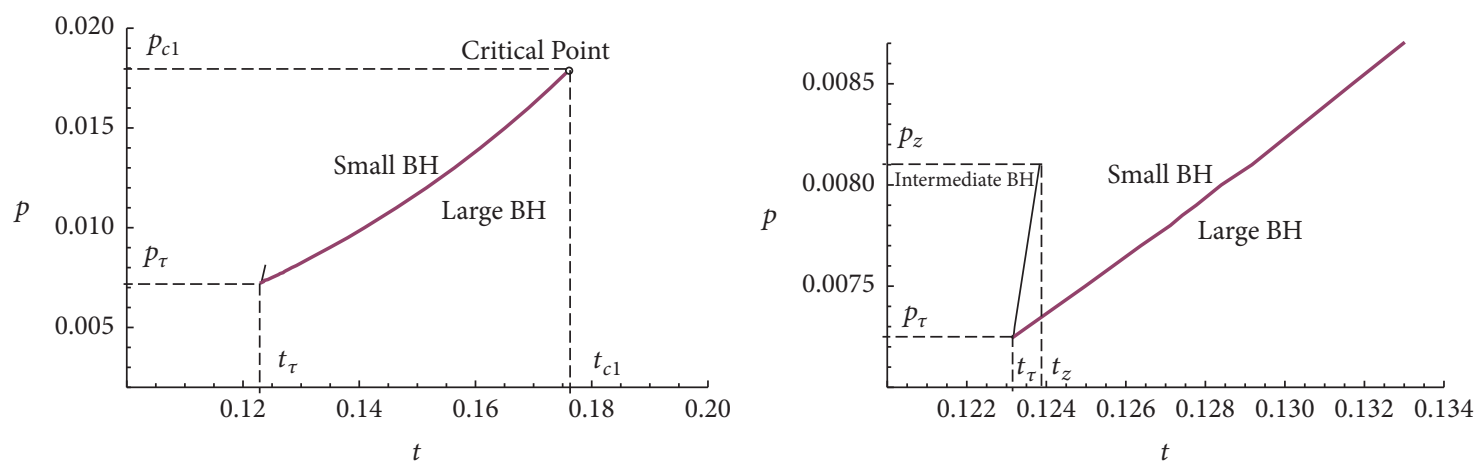

Figure 3: The coexistence line of the VdW-like SBH/LBH phase transition is depicted by a thick solid line. It initiates from the critical point $\left(p_{c 1}, t_{c 1}\right)$ and terminates at $\left(p_{\tau}, t_{\tau}\right)$. The solid line describes the "coexistence line" of small and intermediate black holes, separated by a finite gap in $g$, indicating the zeroth-order phase transition. It commences from $\left(t_{z}, p_{z}\right)$ and terminates at $\left(p_{\tau}, t_{\tau}\right)$.
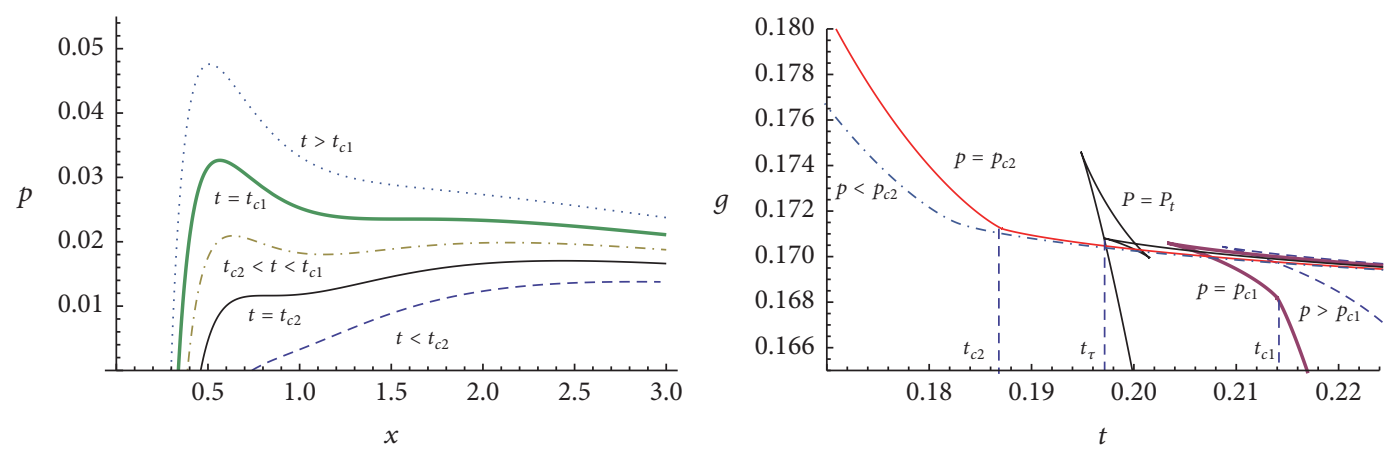

FIGURE 4: Born-Infeld AdS black holes for $d=4$ and $w_{2}=-0.14$. There are two critical points at positive pressure.

For $p<p_{\tau}$ only one phase of large black holes exists. When taking $w_{2}=-0.124$, we obtain

$$
\begin{aligned}
\left(t_{\tau}, t_{z}, t_{c 1}\right) & \approx(0.12316,0.123825,0.175593) \\
\left(p_{\tau}, p_{z}, p_{c 1}\right) & \approx(0.0072472,0.008104,0.0177545)
\end{aligned}
$$

(3) $w_{2} \in(-1 / 2 \pi,-0.132795)$, there exist two critical points with positive pressure, and the similar RPT also occurs. As shown in Figure 4, we obtain

$$
\begin{aligned}
& \left(t_{c 2}, t_{\tau}, t_{z}, t_{c 1}\right) \\
& \quad \approx(0.187113,0.197121,0.198064,0.2139999), \\
& \left(p_{c 2}, p_{\tau}, p_{z}, p_{c 1}\right) \\
& \quad \approx(0.0116313,0.018695,0.0194174,0.0235228),
\end{aligned}
$$$$
\text { when taking } w_{2}=-0.14 \text {. }
$$
by

With regard to $\left|w_{2}\right|>1 / 2 \pi$, the solution of (27) is given

$$
y_{3}=-\cosh \left(\frac{1}{3} \arccos \left(-2 \pi w_{2}\right)\right)
$$

which violates the constraint condition $|y| \leq 1 / \sqrt{2}$.
All in all, when the parameter $w_{2}$ satisfies $-1 / 2 \pi<w_{2}<$ 0 , the Van der Waals-like SBH/LBH phase transition appears. In addition, the interesting RPT happens in case of $-1 / 2 \pi<$ $w_{2}<-1 / \sqrt{8} \pi$.

3.2. $P-V$ Criticality for $d=5$. Then (26) can be rewritten as

$$
\begin{aligned}
F\left(x_{c}\right)= & \frac{1}{x_{c}}\left(x_{c}^{6}+6\right)^{-3 / 2}-\frac{5}{18 x_{c}}\left(x_{c}^{6}+6\right)^{-1 / 2} \\
& -\frac{2 \pi}{27}\left(w_{2}+\frac{3 w_{3}}{x_{c}}\right)=0 .
\end{aligned}
$$

Evidently, it is not possible to obtain analytic solution of above equation. To see more closely the phase transition of the Born-Infeld AdS black hole, here we analyze the asymptotic property of the function $F\left(x_{c}\right)$. In addition, the function $d F\left(x_{c}\right) / d x_{c}$ reads

$$
\begin{aligned}
\frac{d F\left(x_{c}\right)}{d x_{c}}= & 72\left(1-\frac{5 x_{c}^{6}}{24}\right)^{2}+\frac{135 x_{c}^{12}}{8} \\
& +4 \pi w_{3}\left(x_{c}^{6}+6\right)^{5 / 2} .
\end{aligned}
$$

Evidently, (33) has more than one real roots. For different values of $w_{2}$ and $w_{3}$, we will investigate the phase structure and criticality in the extended phase space. 


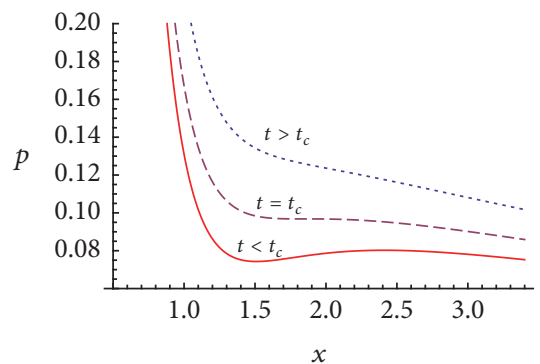

(a)

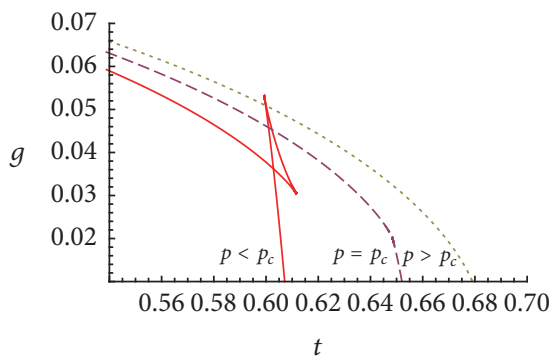

(b)

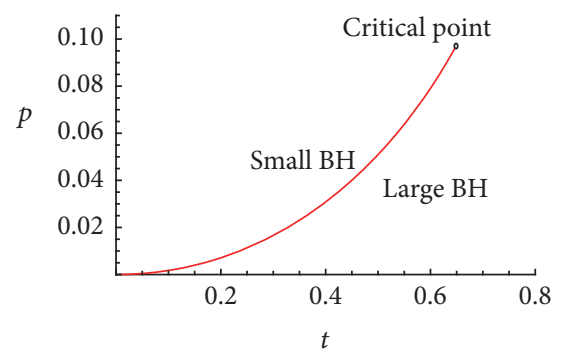

(c)

FIgURE 5: Born-Infeld AdS black holes for $d=5, w_{2}=-0.25$, and $w_{3}=0.1$. (a) The $p-x$ diagram. The dashed curve represents critical isotherm at $t=t_{c}$. The dotted and solid curves correspond to $t>t_{c}$ and $t<t_{c}$, respectively. (b) The $g-t$ diagram. The solid curve represents $p<p_{c}$, the dotted curve correspond to $p>p_{c}$, and the dashed curve is for $p=p_{c}$. We observe standard swallowtail behavior. (c) The $p-t$ diagram, showing the coexistence line of SBH/LBH phase transition terminating at a critical point. These plots are analogous to typical behavior of the liquid-gas phase transition of the VdW fluid.
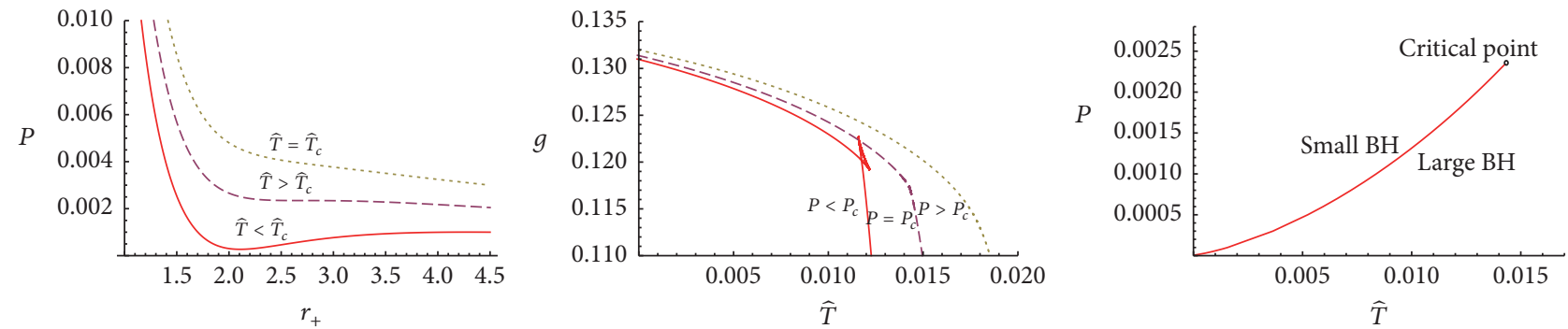

FIGURE 6: Born-Infeld AdS black holes for $d=5, w_{2}=0.001$, and $w_{3}=-0.02$.

3.2.1. $w_{2}>0$ and $w_{3}>0$. When $x_{c} \rightarrow+\infty, F\left(x_{c}\right)$ equals $-2 \pi w_{2} / 27$. Near the origin $x=0$, we have

$$
F\left(x_{c}\right)=-\frac{\left(\sqrt{6}+12 \pi w_{3}\right)}{54 x_{c}}
$$

namely, $F\left(x_{c}\right)$ approaches $-\infty$ on account of $w_{3}>-1 / 2 \sqrt{6} \pi$. Moreover, function $d F\left(x_{c}\right) / d x_{c}$ is always positive, so there is no real solution for $x_{+}$. Therefore, there is no critical point.

3.2.2. $w_{2}<0$ and $w_{3}>0$. Here we adopt similar discussions above. The function $F\left(x_{c}\right)=-2 \pi w_{2} / 27>0$ in case of $w_{2}<0$. However, $F\left(x_{c}\right)$ approaches $-\infty$ near the origin $x=0$. Evidently, there is only one positive root of (33) on account of $d F\left(x_{c}\right) / d x_{c}>0$. Then, a critical point occurs. In Figure 5, we display VdW-like small/large black hole phase transition in the system.

3.2.3. $w_{2}>0$ and $w_{3}<0$. In this case, it is hard work to discuss the asymptotic property of (33). Here we resort to graphical and numerical methods and also find the existence of VdW-like small/large black hole phase transition in the system; see Figure 6.

3.2.4. $w_{2}<0$ and $w_{3}<0$. In [58], Hendi et al. pointed out that the VdW-like SBH/LBH phase transition occurs when $w_{2}<0$ and $w_{3}<0$. Actually, there are some other interesting phase transitions.

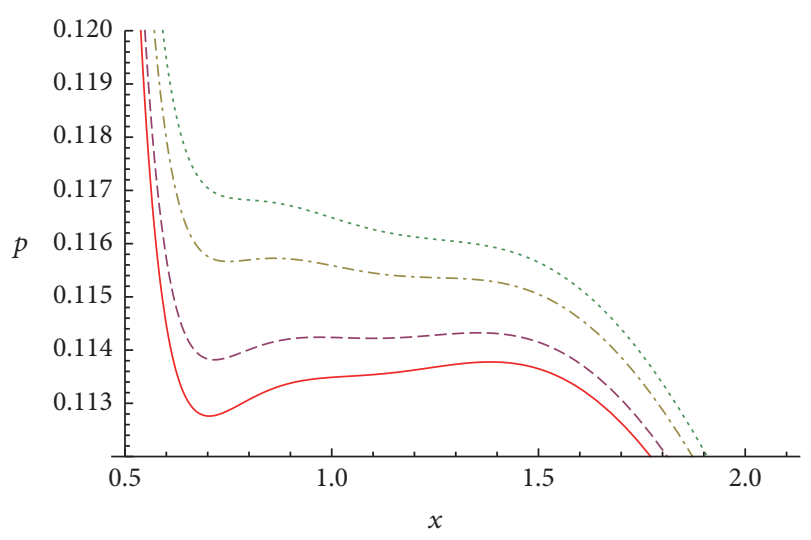

FIgURE 7: Behavior of $p$ as a function of $x$ for $t=$ $0.495,0.497,0.4988$, and 0.5 from bottom to top. For small pressure, one can see that there are stable SBH and LBH branches, which implies the occurrence of VdW-like phase transition. With the increasing of the temperature, there appears a new stable IBH branch. Further increasing the pressure, this branch disappears.

For the case of $w_{2}=-0.084825$ and $w_{3}=-0.045$, the pressure $p$ has three critical points, that is, $\left(p_{c 1}, p_{c 2}\right.$, $\left.p_{c 3}\right)=(0.113983,0.115076,0.116354)$ and $\left(t_{c 1}, t_{c 2}, t_{c 3}\right)=$ $(0.496659,0.498326,0.499503)$. We plot the pressure $p$ as a function of $x$ for $t=0.48,0.497,0.4988$, and 0.51 (from bottom to top) in Figure 7. When $p<p_{c 1}$, there exists a characteristic swallowtail behavior in the $g-t$ diagram, and 


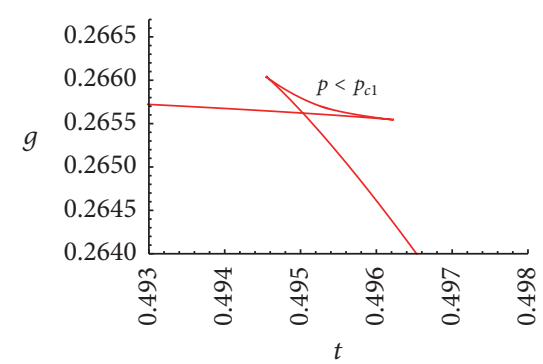

(a)

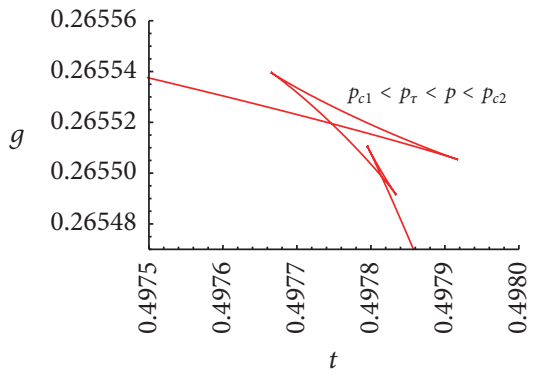

(d)

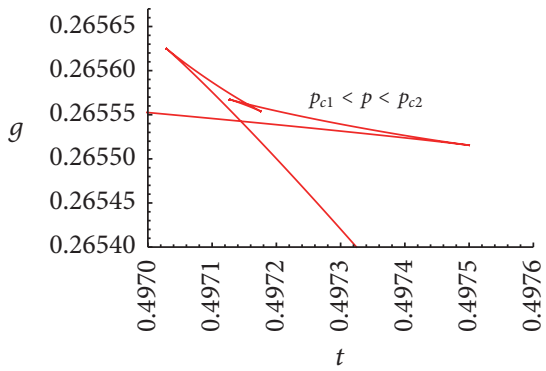

(b)

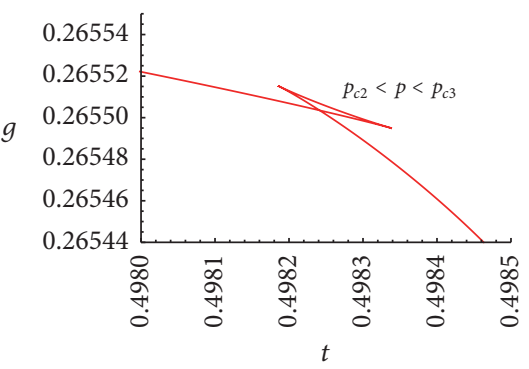

(e)

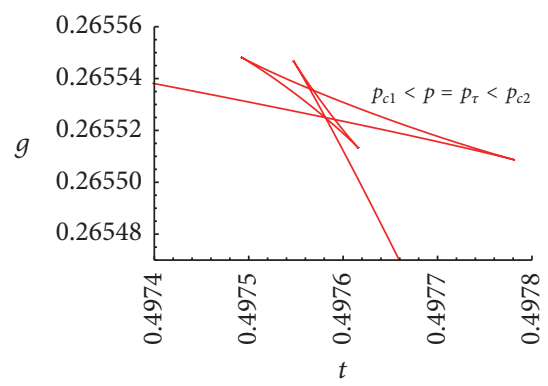

(c)

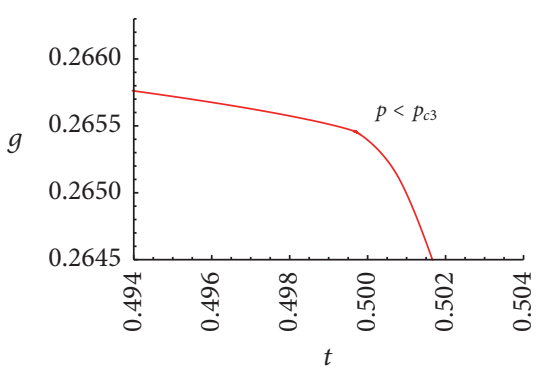

(f)

Figure 8: Gibbs free energy of five-dimensional AdS black holes for $w_{2}=-0.084825$ and $w_{3}=-0.045$.

a VdW-like SBH/LBH phase transition will occur. Further increasing $p$ such that $p_{c 1}<p<p_{c 2}$, there appears a new stable IBH branch. For the corresponding Gibbs free energy in Figure 8, three black hole phases (i.e., small, large, and intermediate black holes) coexist together. Therefore, we observe a triple point characterized by $\left(p_{\tau}, t_{\tau}\right)=$ $(0.01960,0.11226)$. Slightly above this pressure, a standard SBH/IBH/LBH phase transition will appear in the system with the increase of $t$. And such phase transition disappears when $p_{c 2}$ is approached.

Further increasing $p$, the stable IBH branch vanishes in case of $p_{c 2}<p<p_{c 3}$. And only one stable branch survives when $p>p_{c 3}$. In the ranges $p<p_{c 1}$ and $p_{c 2}<p<p_{c 3}$, one characteristic swallowtail behavior is displayed in Figure 8. When $p>p_{c 3}$, there is no such behavior.

\section{Conclusions and Discussions}

In the extended phase space, we have studied the phase transition and critical behavior of topological AdS black holes in the four- and five-dimensional Born-Infeld-massive gravity. For $d=4$, we found that when the horizon topology is spherical $(k=1)$, Ricci flat $(k=0)$, or hyperbolic $(k=-1)$, there always exist the Van der Waals-like SBH/LBH phase transitions when the coupling coefficients of massive potential are located in the region $-1 / 2 \pi<w_{2}<0$. In addition, a monotonic lowering of the temperature yields a large-small-large black hole transition in the region $-1 / 2 \pi<$ $w_{2}<-1 / \sqrt{8} \pi$, where we refer to the former large state as an intermediate black hole (IBH), which is reminiscent of RPTs. Moreover, this process is also accompanied by a discontinuity in the global minimum of the Gibbs free energy, referred to as a zeroth-order phase transition.
In some range of the parameters, there are three critical points for five-dimensional Born-Infeld AdS black hole. In such range, the Gibbs free energy displays the behavior of two swallowtails. This phenomenon has been never recovered before.

Recent observations of gravitational waves have put an upper bound of $1.2 \times 10^{-22} \mathrm{eV} / \mathrm{c}^{2}$ on the graviton's mass [62]. We can find in 4-dimensional case; the interesting RPTs can always appear as long as the parameters $q$ and $b$ take the suitable values with the constant $k$ taking the values \pm 1 . When the constant $k=0$, the role of the graviton's mass is highlighted, the parameters $q$ and $b$ cannot take an acceptable range (means in the framework of the Born-Infeld theory) to make the parameter $w_{2} \in(-1 / 2 \pi,-1 / \sqrt{8} \pi)$, which means only the VdW-like phase transitions might happen. In the 5dimensional case, when the constant $k=1$, this interesting phenomenon could appear as long as the parameters $q$ and $b$ take the suitable values. There are no three critical points when the constant $k$ takes -1 or 0 , because the parameter $w_{2}$ is always positive.

Reference [57] shows that the RPTs only exist in the 4dimensional Born-Infeld AdS black hole with a spherical horizon and also gives the proof that there is no reentrant phase transition in the system of higher $(\geq 5)$ dimensional Born-Infeld AdS black hole. Reference [48] demonstrated that there only exists the Van der Waals-like phase transition in the 4-dimensional AdS black hole in massive gravity with Maxwell's electromagnetic field theory. Our results reveal that the nonlinear electromagnetic field plays an important role in the phase transition of the 4-dimensional AdS black hole, and the massive gravity could bring richer phase structures and critical behavior (triple critical points) than that of the Born-Infeld term in the 5-dimensional AdS black hole. 
Recently, the charged black hole [63], Born-Infeld black hole [64], and black hole in the Maxwell and Yang-Mills fields [65] have been constructed in Gauss-Bonnet-massive gravity. Only Van der Waals-like first-order SBH/LBH phase transition exists in these models. In addition, the RPT and triple points also occur in the higher-dimensional rotating AdS black holes [66, 67] and higher-dimensional GaussBonnet AdS black hole [68-70]. It would be interesting to extend our discussion to these black holes in Gauss-Bonnet and 3rd-order Lovelock-massive gravity.

\section{Conflicts of Interest}

The authors declare that they have no conflicts of interest.

\section{Acknowledgments}

The work is supported by the National Natural Science Foundation of China under Grant nos. 11647050, 11605152, 11675139, and 51575420, Scientific Research Program Funded by Shaanxi Provincial Education Department under Program no. 16JK1394, and Natural Science Foundation of Jiangsu Province under Grant no. BK20160452.

\section{References}

[1] S. N. Gupta, "Gravitation and electromagnetism," Physical Review A: Atomic, Molecular and Optical Physics, vol. 96, no. 6, pp. 1683-1685, 1954.

[2] S. Weinberg, "Photons and gravitons in perturbation theory: Derivation of maxwell's and einstein's equations," Physical Review A: Atomic, Molecular and Optical Physics, vol. 138, no. 4B, pp. B988-B1002, 1965.

[3] R. P. Feynman, F. B. Morinigo, W. G. Wagner, and B. Hatfield, Feynman lectures on gravitation, Addison-Wesley, 1995.

[4] S. Capozziello and M. De Laurentis, "Extended theories of gravity," Physics Reports, vol. 509, no. 4-5, pp. 167-320, 2011.

[5] C. de Rham, "Massive gravity," Living Reviews in Relativity, vol. 17, no. 7, 2014.

[6] M. Fierz and W. Pauli, "On relativistic wave equations for particles of arbitrary spin in an electromagnetic field," Proceedings of the Royal Society A Mathematical, Physical and Engineering Sciences, vol. 173, no. 953, pp. 211-232, 1939.

[7] P. van Nieuwenhuizen, "On ghost-free tensor Lagrangians and linearized gravitation," Nuclear Physics B, vol. 60, no. C, pp. 478492, 1973.

[8] H. van Dam and M. Veltman, "Massive and mass-less YangMills and gravitational fields," Nuclear Physics B, vol. 22, no. 2, pp. 397-411, 1970.

[9] V. I. Zakharov, "Linearized gravitation theory and the graviton mass," JETP Lett, vol. 12, p. 312, 1970.

[10] A. I. Vainshtein, "To the problem of nonvanishing gravitation mass," Physics Letters B, vol. 39, no. 3, pp. 393-394, 1972.

[11] C. de Rham and G. Gabadadze, "Generalization of the FierzPauli action," Physical Review D: Particles, Fields, Gravitation and Cosmology, vol. 82, Article ID 044020, 2010.

[12] C. de Rham, G. Gabadadze, and A. J. Tolley, "Resummation of massive gravity," Physical Review Letters, vol. 106, no. 23, Article ID 231101, 2011.
[13] K. Hinterbichler, "Theoretical aspects of massive gravity," Reviews of Modern Physics, vol. 84, no. 2, 2012.

[14] D. G. Boulware and S. Deser, "Can gravitation have a finite range?" Physical Review D: Particles, Fields, Gravitation and Cosmology, vol. 6, no. 12, pp. 3368-3382, 1972.

[15] D. Vegh, "Holography without translational symmetry," High Energy Physics - Theory, 2013.

[16] A. Adams, D. A. Roberts, and O. Saremi, "Hawking-Page transition in holographic massive gravity," Physical Review D: Particles, Fields, Gravitation and Cosmology, vol. 91, no. 4, 046003, 9 pages, 2015.

[17] T. M. Nieuwenhuizen, "Exact Schwarzschild-de Sitter black holes in a family of massive gravity models," Physical Review D: Particles, Fields, Gravitation and Cosmology, vol. 84, no. 2, Article ID 024038, 2011.

[18] R. Brito, V. Cardoso, and P. Pani, "Black holes with massive graviton hair," Physical Review D: Particles, Fields, Gravitation and Cosmology, vol. 88, no. 6, Article ID 064006, 2013.

[19] S. G. Ghosh, L. Tannukij, and P. Wongjun, "A class of black holes in dRGT massive gravity and their thermodynamical properties," The European Physical Journal C, vol. 76, no. 3, article no. 119, 2016.

[20] L. Berezhiani, G. Chkareuli, C. De Rham, G. Gabadadze, and A. J. Tolley, "On black holes in massive gravity," Physical Review D: Particles, Fields, Gravitation and Cosmology, vol. 85, no. 4, Article ID 044024, 2012.

[21] R.-G. Cai, Y.-P. Hu, Q.-Y. Pan, and Y.-L. Zhang, "Thermodynamics of black holes in massive gravity," Physical Review D: Particles, Fields, Gravitation and Cosmology, vol. 91, no. 2, 024032, 12 pages, 2015.

[22] B. P. Dolan, "Pressure and volume in the first law of black hole thermodynamics," Classical and Quantum Gravity, vol. 28, no. 23, Article ID 235017, 2011.

[23] B. P. Dolan, "The cosmological constant and black-hole thermodynamic potentials," Classical and Quantum Gravity, vol. 28, no. 12, Article ID 125020, 2011.

[24] D. Kubiznak and R. B. Mann, "P-V criticality of charged AdS black holes," Journal of High Energy Physics, vol. 1207, no. 33, 2012.

[25] D. Hansen, D. Kubiznak, and R. B. Mann, "Universality of $P-V$ criticality in horizon thermodynamics," Journal of High Energy Physics, vol. 1701, no. 47, 2017.

[26] R.-G. Cai, L.-M. Cao, L. Li, and R.-Q. Yang, "P-V criticality in the extended phase space of Gauss-Bonnet black holes in AdS space," Journal of High Energy Physics, vol. 2013, no. 9, article no. 005, 2013.

[27] S. Dutta, A. Jain, and R. Soni, "Dyonic black hole and holography," Journal of High Energy Physics, vol. 2013, no. 12, 2013.

[28] S. H. Hendi and M. H. Vahidinia, "Extended phase space thermodynamics and $P-V$ criticality of black holes with a nonlinear source," Physical Review D: Particles, Fields, Gravitation and Cosmology, vol. 88, no. 8, Article ID 084045, 11 pages, 2013.

[29] W. Xu, H. Xu, and L. Zhao, "Gauss-bonnet coupling constant as a free thermodynamical variable and the associated criticality," The European Physical Journal C, vol. 74, no. 7, article 2970, 2014.

[30] H.-H. Zhao, L.-C. Zhang, M.-S. Ma, and R. Zhao, " $P$ - $V$ criticality of higher dimensional charged topological dilaton de Sitter black holes," Physical Review D: Particles, Fields, Gravitation and Cosmology, vol. 90, no. 6, Article ID 064018, 2014. 
[31] D. C. Zou, Y. Liu, and B. Wang, "Critical behavior of charged Gauss-Bonnet-AdS black holes in the grand canonical ensemble," Physical Review D: Particles, Fields, Gravitation and Cosmology, vol. 90, no. 4, Article ID 044063, 2014.

[32] H. Xu, W. Xu, and L. Zhao, "Extended phase space thermodynamics for third-order Lovelock black holes in diverse dimensions," The European Physical Journal C, vol. 74, article 3074, 2014.

[33] W. Xu and L. Zhao, "Critical phenomena of static charged AdS black holes in conformal gravity," Physics Letters. B. Particle Physics, Nuclear Physics and Cosmology, vol. 736, pp. 214-220, 2014.

[34] M. H. Dehghani, S. Kamrani, and A. Sheykhi, "P-V criticality of charged dilatonic black holes," Physical Review D: Particles, Fields, Gravitation and Cosmology, vol. 90, no. 10, Article ID 104020, 2014.

[35] C. O. Lee, "The extended thermodynamic properties of TaubNUT/Bolt-AdS spaces," Physics Letters. B. Particle Physics, Nuclear Physics and Cosmology, vol. 738, pp. 294-300, 2014.

[36] J. L. Zhang, R. G. Cai, and H. Yu, "Phase transition and thermodynamical geometry for Schwarzschild AdS black hole in $\mathrm{AdS}_{5} \times \mathrm{S}_{5}$ spacetime," Journal of High Energy Physics, vol. 2015, no. 2, article 143, 2015.

[37] M. Zhang, Z.-Y. Yang, D.-C. Zou, W. Xu, and R.-H. Yue, "P $V$ criticality of AdS black hole in the Einstein-Maxwell-powerYang-MILls gravity," General Relativity and Gravitation, vol. 47, no. 2, 2015.

[38] Y. Liu, D.-C. Zou, and B. Wang, "Signature of the Van der Waals like small-large charged AdS black hole phase transition in quasinormal modes," Journal of High Energy Physics, vol. 2014, no. 9, article no. 179, 2014.

[39] J. L. Zhang, R. G. Cai, and H. Yu, "Phase transition and thermodynamical geometry of Reissner-Nordström-AdS black holes in extended phase space," Physical Review D: Particles, Fields, Gravitation and Cosmology, vol. 91, no. 4, Article ID 044028, 14 pages, 2015.

[40] B. R. Majhi and S. Samanta, "P-V criticality of AdS black holes in a general framework," Physics Letters. B. Particle Physics, Nuclear Physics and Cosmology, vol. 773, pp. 203-207, 2017.

[41] S. H. Hendi, B. E. Panah, S. Panahiyan, and M. S. Talezadeh, "Geometrical thermodynamics and P-V criticality of the black holes with power-law Maxwell field," The European Physical Journal C, vol. 77, no. 2, article no. 133, 2017.

[42] S. H. Hendi, S. Panahiyan, B. Eslam Panah, M. Faizal, and M. Momennia, "Critical behavior of charged black holes in GaussBonnet gravity's rainbow," Physical Review D: Particles, Fields, Gravitation and Cosmology, vol. 94, no. 2, Article ID 024028, 2016.

[43] D. Kubiznak, R. B. Mann, and M. Teo, "Black hole chemistry: thermodynamics with lambda," Classical and Quantum Gravity, vol. 34, no. 6, 2017.

[44] X. Kuang and O. Miskovic, "Thermal phase transitions of dimensionally continued AdS black holes," Physical Review D: Particles, Fields, Gravitation and Cosmology, vol. 95, no. 4, 2017.

[45] Y. G. Miao and Y. M. Wu, "Thermodynamics of the Schwarzschild-AdS black hole with a minimal length," Advances in High Energy Physics, vol. 2017, 14 pages, 2017.

[46] M. Cadoni, E. Franzin, and M. Tuveri, "Van der Waals-like behaviour of charged black holes and hysteresis in the dual QFTs," Physics Letters. B. Particle Physics, Nuclear Physics and Cosmology, vol. 768, pp. 393-396, 2017.
[47] S. H. Hendi, B. Eslam Panah, and S. Panahiyan, "EinsteinBorn-Infeld-massive gravity: adS-black hole solutions and their thermodynamical properties," Journal of High Energy Physics, vol. 95, no. 11, 2015.

[48] J. Xu, L. M. Cao, and Y. P. Hu, "P-V criticality in the extended phase space of black holes in massive gravity," Phys. Rev. D, vol. 91, Article ID 124033, 2015.

[49] M. Zhang and W. B. Liu, "Coexistent physics of massive black holes in the phase transitions," General Relativity and Quantum Cosmology, 2016.

[50] S. H. Hendi, S. Panahiyan, B. Eslam Panah, and M. Momennia, "Phase transition of charged black holes in massive gravity through new methods," Annalen der Physik, vol. 528, no. 11-12, pp. 819-834, 2016.

[51] D. Zou, Y. Liu, and R. Yue, "Behavior of quasinormal modes and Van der Waals-like phase transition of charged AdS black holes in massive gravity," The European Physical Journal C, vol. 77, no. 6, 2017.

[52] D. Zou, R. Yue, and M. Zhang, "Reentrant phase transitions of higher-dimensional AdS black holes in dRGT massive gravity," The European Physical Journal C, vol. 77, no. 4, 2017.

[53] T. Narayanan and A. Kumar, "Reentrant phase transitions in multicomponent liquid mixtures," Physics Reports, vol. 249, no. 3, pp. 135-218, 1994.

[54] M. Born and L. Infeld, "Foundations of the new field theory," Proceedings of the Royal Society A Mathematical, Physical and Engineering Sciences, vol. 144, no. 852, pp. 425-451, 1934.

[55] B. Hoffmann, "Gravitational and electromagnetic mass in the born-infeld electrodynamics," Physical Review A: Atomic, Molecular and Optical Physics, vol. 47, no. 11, pp. 877-880, 1935.

[56] S. Gunasekaran, D. Kubiznak, and R. B. Mann, "Extended phase space thermodynamics for charged and rotating black holes and Born-Infeld vacuum polarization," Journal of High Energy Physics, vol. 2012, no. 11, article 110, 2012.

[57] D.-C. Zou, S.-J. Zhang, and B. Wang, "Critical behavior of BornInfeld AdS black holes in the extended phase space thermodynamics," Physical Review D: Particles, Fields, Gravitation and Cosmology, vol. 89, Article ID 044002, 2014.

[58] S. H. Hendi, B. E. Panah, and S. Panahiyan, "Einstein-BornInfeld-massive gravity: adS-black hole solutions and their thermodynamical properties," Journal of High Energy Physics, vol. 2015, no. 11, article no. 157, pp. 1-29, 2015.

[59] D. Kastor, S. Ray, and J. Traschen, "Enthalpy and the mechanics of AdS black holes," Classical and Quantum Gravity, vol. 26, no. 19, Article ID 195011, 195011, 16 pages, 2009.

[60] M. Cvetic, G. W. Gibbons, D. Kubiznak, and C. N. Pope, "Black Hole Enthalpy and an Entropy Inequality for the Thermodynamic Volume," Phys. Rev. D, vol. 84, Article ID 024037, 2011.

[61] V. P. Maslov, "Zeroth-order phase transitions," Mathematical Notes, vol. 76, no. 5-6, pp. 697-710, 2004.

[62] B. P. Abbott et al., "Observation of gravitational waves from a binary black hole merger," Physical Review Letters, vol. 116, no. 6, 2016.

[63] S. H. Hendi, S. Panahiyan, and B. E. Panah, "Charged black hole solutions in Gauss-Bonnet-massive gravity," Journal of High Energy Physics, vol. 2016, no. 1, article 129, pp. 1-35, 2016.

[64] S. H. Hendi, G.-Q. Li, J.-X. Mo, S. Panahiyan, and B. E. Panah, "New perspective for black hole thermodynamics in GaussBonnet-Born-Infeld massive gravity," The European Physical Journal C, vol. 76, no. 10, article 571, 2016. 
[65] K. Meng and J. Li, "Black hole solution of Gauss-Bonnet massive gravity coupled to Maxwell and Yang-Mills fields in five dimensions," EPL (Europhysics Letters), vol. 116, no. 1, Article ID 10005, 2016.

[66] N. Altamirano, D. Kubiznak, and R. Mann, "Reentrant phase transitions in rotating AdS black holes," Physical Review D: Particles, Fields, Gravitation and Cosmology, vol. 88, no. 10, Article ID 101502, 5 pages, 2013.

[67] N. Altamirano, D. Kubiznak, R. B. Mann, and Z. Sherkatghanad, "Thermodynamics of rotating black holes and black rings: phase transitions and thermodynamic volume," Galaxies, vol. 2, no. 89, 2014.

[68] S.-W. Wei and Y.-X. Liu, "Triple points and phase diagrams in the extended phase space of charged Gauss-Bonnet black holes in AdS space," Physical Review D: Particles, Fields, Gravitation and Cosmology, vol. 90, no. 4, Article ID 044057, 2014.

[69] A. M. Frassino, D. Kubizňák, R. B. Mann, and F. Simovic, "Multiple reentrant phase transitions and triple points in Lovelock thermodynamics," Journal of High Energy Physics, vol. 2014, no. 9, article no. 80, pp. 1-47, 2014.

[70] R. A. Hennigar, E. Tjoa, and R. B. Mann, "Thermodynamics of hairy black holes in Lovelock gravity," Journal of High Energy Physics, vol. 2017, no. 2, article 70, 2017. 

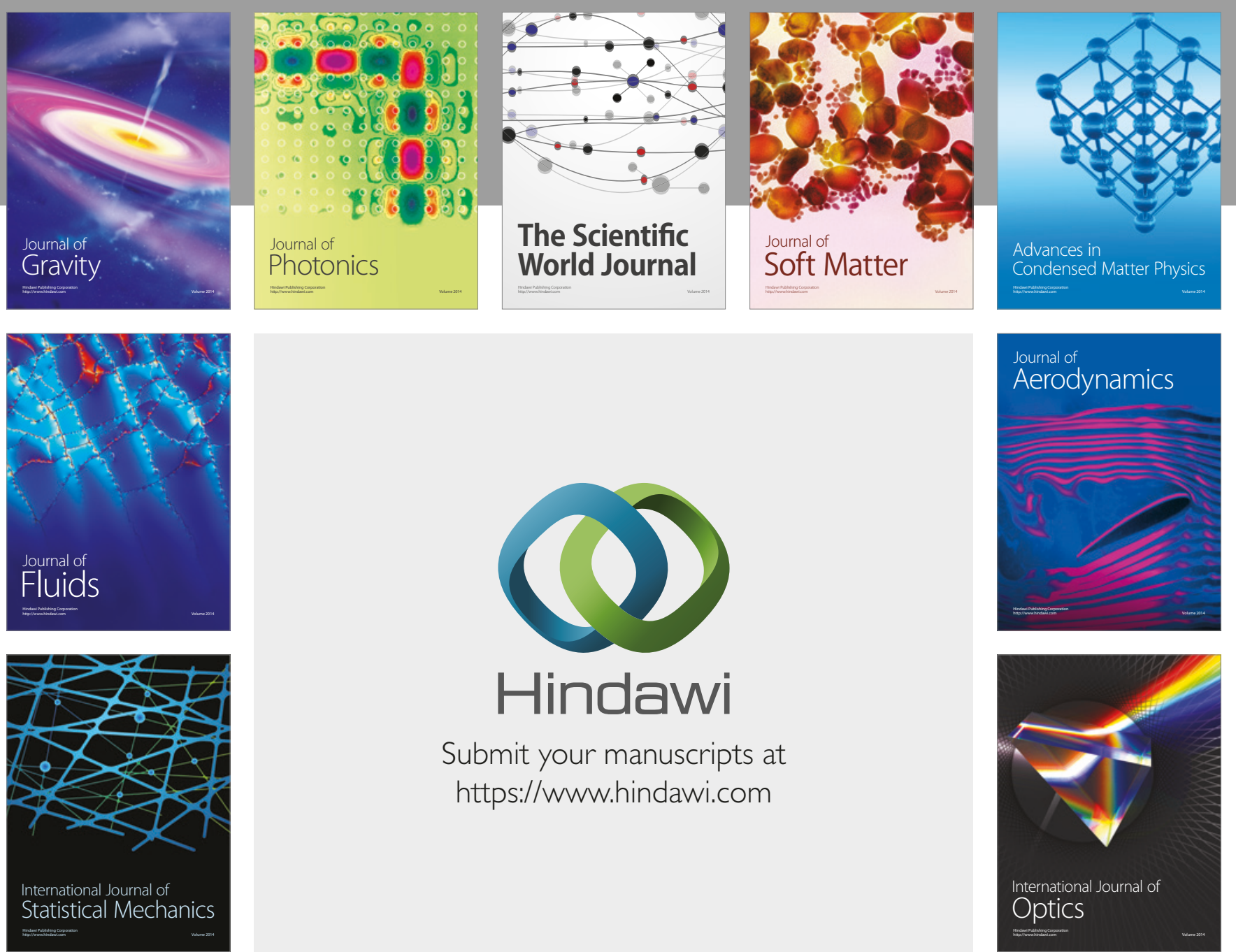

Submit your manuscripts at

https://www.hindawi.com
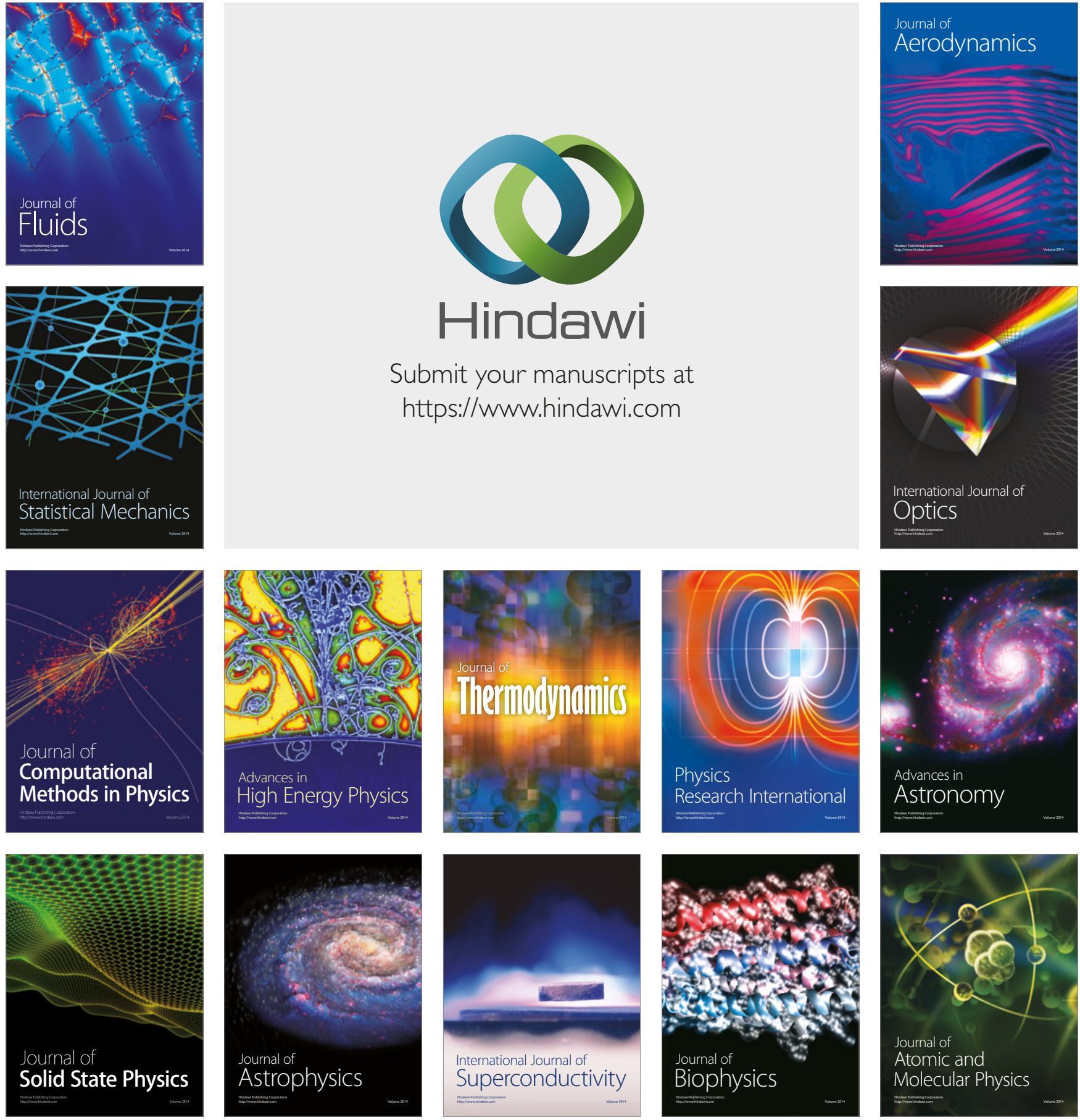